J. Clin. Chem. Clin. Biochem.

Vol. 18, 1980, pp. 233-236

\title{
Die Bestimmung des kolloidosmotischen Drucks aus der Plasmadichte mittels der Biegeschwingermethode ${ }^{1}$ )
}

\author{
Von M. Moser, H. Hinghofer-Szalkay, Th. Kenner und H. Holzer \\ Aus dem Physiologischen Institut (Vorstand: Prof. Dr. Th. Kenner) und der Medizinischen Universitätsklinik \\ (Vorstand: Prof. Dr. S. Sailer) der Universität Graz
}

(Eingegangen am 9. Juli/12. November 1979)

Zusammenfassung: Der kolloidosmotische Druck im Blutplasma ist eine Zustandsvariable von großer klinischer und physiologischer Bedeutung. Seine Größe ist, ebenso wie die Dichte des Plasmas, von der Gesamteiweißkonzentration abhängig. Daher läßt sich aus der Plasmadichte der kolloidosmotische Druck berechnen, wobei Abweichungen vom gemessenen Wert auf unterschiedlichen Gehalt an Albumin und Globulin sowie an niedermolekularen Plasmainhaltsstoffen zurückzuführen sind.

Die direkte Messung des kolloidosmotischen Druckes ist zeitraubend und aufwendig. Die Biegeschwingermethode ermöglicht hingegen eine einfache und schnelle Präzisionsmessung der Dichte. Aufgrund unserer Resultate konnten wir eine Gleichung zur Kalkulation des kolloidosmotischen Druckes aus der Dichte aufstellen. Das für eine Bestimmung erforderliche Probenvolumen beträgt 0,2 ml, die Meßzeit einige Sekunden.

\section{Measurement of the colloid osmotic pressure from plasma density by means of the mechanical oscillator technique}

Summary: The colloid osmotic pressure of plasma is a clinically and physiologically important variable. Like the plasma density, it is a function of the total concentration of plasma proteins. Thus, plasma colloid osmotic pressure can be determined from measurement of the plasma density. Minor variations are caused by variations of the albuminglobulin ratio and, much less frequently, by increased concentration of low molecular substances in the plasma.

The direct measurement of the colloid osmotic pressure is a time consuming and complex procedure. The determination of the plasma density using the mechanical oscillator technique is a simple and fast procedure. From our results we were able to derive and prove the applicability of an equation which permits the calculation of the colloid osmotic pressure from the measured values of the plasma density. The plasma volume needed for the measurement is $0.2 \mathrm{ml}$, the measuring time amounts to a few seconds.

\section{Einführung}

Die Bedeutung des kolloidosmotischen Druckes für die Ėinstellung des Flüssigkeitsgleichgewichtes zwischen Plasma und Iñterstitium ist seit Ende des vorigen Jahrhunderts bekannt (12). In der Literatur finden sich die verschịedenșten Versuche, den kolloidosmotischen Druck zu messen oder indirekt zu bestimmen. Trotż beträchtlicher Vereinfachung der Messung durch elektronische Manometer $(1,7)$ sind die heute üblichen Meßzeiten noch in der Größenordnung von 2 bis 15 Minuten (Hansen-Osmometer) und auch Meßvorgang und -vor-

1) Unterstützt durch den Fonḍ̣ zur Förderung der wisssenșçhaftlichen Forschung. bereitung sind umständlich und zeitraubend. Eine ausführliche Zusammenfassung der meßtechnischen Schwierigkeiten ist bei Intaglietta \& Zweifach gegeben (3).

Aụs diesen Gründen wurde wiederholt versucht, den kolloidosmotischen Druck aus einfacher zu erhaltenden Meßgrößen indirekt zu bestimmen. Dazu wurden unter anderem der Plasmaeiweißgehalt $(5,6)$, der Refraktionsindex (10) und die Plasmadichte (11) herangezogen.

Mit einer neuen Dichtemeßmethode nach Kratky, Leopold \& Stabinger (4) kann die Dichte von kleinen Probenvolumina in kurzer Zeit mit einer Genauigkeit von $10^{-6} \mathrm{~kg} / \mathrm{l}$ gemessen werden. Es ergibt sich die Möglichkeit, die Relation zwischen kolloidosmotischem Druck 
und Plasmadichte zur schnellen indirekten Bestimmung des kolloidosmotischen Druckes anzuwenden. Dieses Verfahren ist Gegenstand der vorliegenden Untersuchung.
Tab. 1. Ubersicht über unsere Ergebnisse. Zwei: Plasmaproben wurden mit Ultrafiltrat verdünnt, um Relationen in niedrigen Konzentrationsbereichen zu eihalten. In der untersten Zeile ist die Dichte von Ultrafiltrat angegeben.

\section{Methodik}

Von 27 Patienten wurde im Liegen nach Stauung des Oberarmes Blut aus der Kubitalvene abgenommen und kurze Zeit später zentrifugiert. Die Blutproben wurden in sterilen Einmalspritzen verwahrt, in die vorher $20 \mu \mathrm{l}$ Heparinlösung gesaugt wurde. Nach Zentrifugation ( $20 \mathrm{~min}$ bei 5000 Umdrehungen pro Minute bei $20^{\circ} \mathrm{C}$ ) wurde das überstehende Plasma in Einmalspritzen abgesaugt und die Dichte bei $20^{\circ} \mathrm{C}$ sowie der kolloidosmotische Druck bei $37^{\circ} \mathrm{C}$ bestimmt.

Zur Herstellung einer Verdünnungsreihe wurde Plasma mit dem Ultrafiltrat der gleichen Plasmaprobe verdünnt. Das Ultrafiltrat wurde mittels Druckfiltration durch eine PM 10-Membran (Amicon) gewonnen. Die Dichten der verdünnten Plasmaproben wurden ebenfalls bei $20,0^{\circ} \mathrm{C}$ gemessen.

Zur Dichtemessung verwendeten wir eine Dichtemeßzelle DMA 602 W der Fa. Paar K.G., Graz. Dieses Gerät arbeitet nach der Biegeschwingermethode (4). Ein stimmgabelähnliches Glas-U-Rohr, dessen Innenvolumen hier $0,1 \mathrm{ml}$ beträgt, wird mit der Probe gefüllt und die Schwingungsfrequenz dieses Schwingkörpers fortlaufend gemessen. Die Elektronik des Gerätes ermittelt daraus nach vorhergehender Kalibrierung die entsprechende Dichte, wobei die Meß- und Rechenzeit für einen Schritt etwa 0,9 Sekunden beträgt und die Digitalanzeige eine Auflösung von $10^{-5} \mathrm{~kg} / \mathrm{l}$ erlaubt. Eine genauere Beschreibung der Meßmethodik und Untersuchungen über ihre Genauigkeit findet sich in 1.c. (4). Jeder Plasmadichtewert war in unseren Messungen auf $10^{-5} \mathrm{~kg} / 1$ reproduzierbar und die Meßwertanzeige in allen Fällen stabil. Eine Beschreibung des Meßprinzips findet sich in 1.c. (2) und l.c. (4).

Die Messung des kolloidosmotischen Druckes erfolgte mittels eines Hansen-Membranosmometers der Fa. Knauer mit thermostatisiertem Probenraum. Als Membran wurde, wie auch zur Ultrazentrifugation, die Type PM 10 von Amicon verwendet. Jeweils vor den Messungen wurde sie 12 Stunden in destilliertes Wasser gelegt und anschließend in physiologischer $\mathrm{NaCl}-$ Lösung erhitzt, um Luftblasen zu entfernen. Als Vergleichslösung für den Meßvorgang diente physiologische $\mathrm{NaCl}-\mathrm{Lösung}$, mit der nach jeder Messung etwa acht Minuten gespült wurde. Vor Beginn jeder Messung wurde eine Spülung mit Probenlösung vorgenommen. Die gemessenen Druckwerte wurden von einem Linseis-Kompensationsschreiber aufgezeichnet. Als Meßwert für den kolloidosmotischen Druck wurde jener Wert verwendet, der sich nach 3 Minuten eingestellt hat. Jede Probe wurde zweimal gemessen.

Das Albumin-Globulinverhältnis wurde mittels Celluloseacetatfolien-Hochspannungselektrophorese bestimmt.

\section{Ergebnisse}

In Tabelle 1 und in Abbildung 1 sind die Ergebnisse unserer Messungen zusammengefaßt. Man erkennt einen nichtlinearen Zusammenhang zwischen Dichte und kolloidosmotischem Druck von Plasma. Unsere Messungen bestätigen die Ergebnisse von Soto-Rivera (11), und wir paßten an unsere Daten die dort beschriebene Gleichung

kolloidosmotischer Druck $=\frac{1,97 \mathrm{~K}\left(\rho_{\mathrm{p}}-\rho_{0}\right)}{1-0,071 \mathrm{~K}\left(\rho_{\mathrm{p}}-\rho_{0}\right)}($ Gl. 1$)$

\begin{tabular}{|c|c|c|c|c|c|}
\hline Name & $\begin{array}{l}\text { Plasma- } \\
\text { dichte } \\
\text { gemessen } \\
\left(20^{\circ} \mathrm{C}\right) \\
\rho(\mathrm{kg} / \mathrm{l})\end{array}$ & \multicolumn{2}{|c|}{$\begin{array}{l}\text { Kolloid- } \\
\text { osmotischer Druck } \\
\text { gemessen aus } \rho \text { be- } \\
\left(37^{\circ} \mathrm{C}\right) \text { rechnet } \\
p \quad p \\
p \quad p \\
(\mathrm{~cm} \quad(\mathrm{~cm} \\
\left.\left.\mathrm{H}_{2} \mathrm{O}\right) \quad \mathrm{H}_{2} \mathrm{O}\right)\end{array}$} & $\frac{\text { Albumin }}{\text { G̣lobulï }}$ & $\begin{array}{l}\text { Bemer- } \\
\text { kungen }\end{array}$ \\
\hline $\begin{array}{l}\text { Pr. } \\
\text { Sö. } \\
\text { Ko. } \\
\text { Ke. } \\
\text { Il. } \\
\text { Ha. } \\
\text { Kl. } \\
\text { Sc. } \\
\text { Da. } \\
\text { Wi.. } \\
\text { Ne. } \\
\text { Ma. } \\
\text { Za. } \\
\text { Le. } \\
\text { Ho. } \\
\text { Ma. } \\
\text { Sc. } \\
\text { Jo. } \\
\text { Mü. } \\
\text { Ra. } \\
\text { Zö. } \\
\text { Kn. } \\
\text { Go. } \\
\text { Sc. } \\
\text { Ko. } \\
\text { Ho. } \\
\text { Gr. }\end{array}$ & $\begin{array}{l}1,02174 \\
1,02705 \\
1,02658 \\
1,02564 \\
1,02269 \\
1,02358 \\
1,02668 \\
1,02549 \\
1,02345 \\
1,02545 \\
1,02653 \\
1,02465 \\
1,02319 \\
1,02870 \\
1,02532 \\
1,02741 \\
1,02709 \\
1,02882 \\
1,02306 \\
1,02751 \\
1,02400 \\
1,02816 \\
1,02754 \\
1,02608 \\
1,02605 \\
1,02530 \\
1,02387 \\
1,01909 \\
1,01684 \\
1,01401 \\
1,01044 \\
1,01475 \\
1,01298 \\
1,01081 \\
1,00810 \\
1,00543\end{array}$ & $\begin{array}{r}21,1 \\
48,2 \\
39,7 \\
41,7 \\
31,3 \\
34,9 \\
45,6 \\
43,4 \\
33,1 \\
38,4 \\
40,0 \\
32,7 \\
35,6 \\
48,8 \\
37,3 \\
44,0 \\
44,4 \\
62,6 \\
36,2 \\
53,2 \\
36,8 \\
48,8 \\
49,5 \\
41,8 \\
43,7 \\
43,5 \\
35,8 \\
23,6 \\
18,6 \\
12,8 \\
6,8 \\
10,3 \\
8,0 \\
5,5 \\
2,9 \\
0\end{array}$ & $\begin{array}{r}26,8 \\
46,6 \\
44,3 \\
40,2 \\
29,6 \\
32,5 \\
44,8 \\
39,6 \\
32,0 \\
39,4 \\
44,1 \\
36,3 \\
31,2 \\
55,5 \\
38,9 \\
48,4 \\
46,8 \\
56,3 \\
30,7 \\
49,0 \\
38,9 \\
52,4 \\
49,1 \\
42,1 \\
42,0 \\
39,0 \\
33,5 \\
20,0 \\
15,3 \\
10,5 \\
5,5 \\
11,6 \\
8,9 \\
5,9 \\
2,7 \\
0 \\
.\end{array}$ & $\begin{array}{l}0,70 \\
0,86 \\
0,87 \\
0,88 \\
0,91 \\
0,95 \\
1,02 \\
1,04 \\
1,12 \\
1,13 \\
1,16 \\
1,27 \\
1,28 \\
1,29 \\
1,31 \\
1,36 \\
1,38 \\
1,46 \\
1,50 \\
1,54 \\
1,58 \\
1,61 \\
1,63 \\
1,72 \\
1,75 \\
1,84 \\
2,14 \\
1 \\
\}\end{array}$ & $\begin{array}{l}\text { Plasma } \\
\text { Kn. } \\
\text { verdünnt } \\
\text { Plasma } \\
\text { Pr. } \\
\text { verdünnt } \\
\text { Ultra- } \\
\text { filtrat }\end{array}$ \\
\hline
\end{tabular}

an, wobei hier $\rho_{\mathrm{p}}$ die Plasmadichte und $\rho_{0}$ die Dichte von Ultrafiltrat bedeuten. Die Konstante $\mathrm{K}$ ist dabei von den Daten abhängig und wurde von uns durch Minimierung des quadratischen Fehlers bestimmt. Die übrigen Zahlen ergeben sich aus einer von Scatchard verwendeten Gleichung bei $\mathrm{pH}=7,4(8)$. Die Summe der quadratischen Fehler ist für unsere Ergebnisse bei $\mathrm{K}=365$ am geringsten (Abbildung 1, durchgezogene Anpassungslinie). Dabei wurdé als $\rho_{0}$ die bei zwei Messungen bestímmte Ultrafiltratdichte $\left(1,00543 \mathrm{~kg} / 1\right.$ bei $\left.20,0{ }^{\circ} \mathrm{C}\right)$ verwendet. Der von Soto-Rivera gefundenen Beziehung zwischen kolloidosmotischem Druck und Dichte entspricht der Wert $\mathrm{K}=353$; sie ist in Abbildung 1 strichliert ein'gezeichnet. 


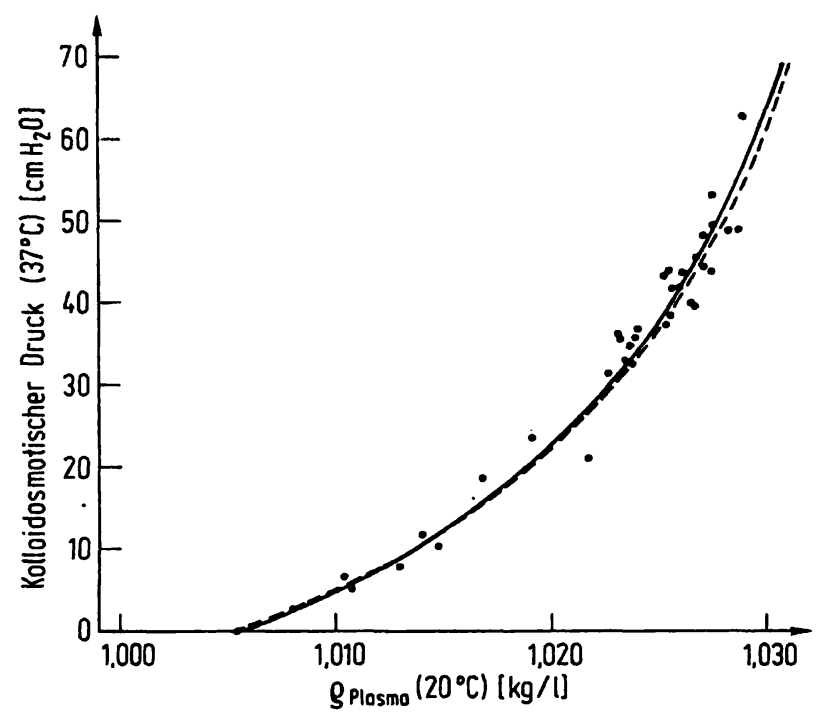

Abb. 1. Relation des kolloidosmotischen Drucks bei $37^{\circ} \mathrm{C}$ $\left(1 \mathrm{~cm} \mathrm{H}_{2} \mathrm{O}=0,098 \mathrm{kPa}\right.$ ) zur Plasmadichte $\left(\rho_{\mathrm{p}}\right)$ bei $20^{\circ} \mathrm{C}$.

Strichliert: Beziehung nach Soto-Rivera (11); durchgezogen: Von uns gefundene Beziehung. Die kleinste Summe der quadratischen Fehler ergab sich nach unseren Messungen bei einem Wert für K von 365 (vgl. Ergebnisse und Diskussion).

\section{Diskussion}

Wie aus der Literatur bekannt (9) und von unserer Arbeitsgruppe bestätigt ist (2), kann der Plasmaeiweißgehalt aus der Plasmadichte berechnet werden. Dies ermöglicht einen Vergleich unserer Ergebnisse mit denen anderer Autoren, die aus der Plasmaeiweißkonzentration auf den kolloidosmotischen Druck schließen. Eine Berechnung des Plasmaeiweißgehaltes aus der Dichte nach (2) und daraus des kolloidosmotischen Druckes nach 1.c. (5) und l.c. (6) lieferte Ergebnisse, die mit unseren Messungen übereinstimmen. Nur im höheren kolloidosmotischen Druckbereich (über $40 \mathrm{~cm} \mathrm{H}_{2} \mathrm{O}$ ) weichen die so berechneten Werte für den kolloidosmotischen Druck von den gemessenen nach unten ab.

Die Berechnung des kolloịdosmotischen Druckes aus der Dichte kann nach Gleichung 1 durchgeführt werden, die vereinfacht werden kann auf:

$$
\begin{aligned}
& \text { kolloidosmotischer } \\
& \text { Druck }
\end{aligned}=\frac{27,7465 \rho_{\mathrm{p}}-27,8971}{1,0439-\rho_{\mathrm{p}}}(\mathrm{mm} \mathrm{Hg})
$$

bzw.

$$
\begin{aligned}
\begin{array}{l}
\text { kolloidosmotischer } \\
\text { Druck }
\end{array}= & \frac{36,5085 \rho_{\mathrm{p}}-36,7068}{1,0439-\rho_{\mathrm{p}}}\left(\mathrm{cm} \mathrm{H}_{2} \mathrm{O}\right) \\
& \left(1 \mathrm{~cm} \mathrm{H}_{2} \mathrm{O}=0,098 \mathrm{kPa}\right)
\end{aligned}
$$

Die bei $20,0^{\circ} \mathrm{C}$ gemessene Dichte, in der Dimension $\mathrm{kg} / \mathrm{l}$ in die Gleichung eingesetzt, ergibt den kolloidosmotischen Druck bei $37,0^{\circ} \mathrm{C}$. Diese Temperaturen wurden aus praktischen Gründen gewählt, da $20^{\circ} \mathrm{C}$ der Raumtemperatur entspricht und $37^{\circ} \mathrm{C}$ derjenigen, bei welcher der kolloidosmotische Druck im Körper wirkt.

Als mittlerer Fehler ergab sich bei unseren Messungen des kolloidosmotischen Drucks eine Differenz von $3,6 \mathrm{~cm}$ $\mathrm{H}_{2} \mathrm{O}$ bzw. $2,7 \mathrm{~mm} \mathrm{Hg}$ zwischen berechnetem und gemessenem Wert, entsprechend 8,7\% des Mittelwerts. Der maximale Fehler betrug $6,7 \mathrm{~cm} \mathrm{H}_{2} \mathrm{O}$ bzw. $4,9 \mathrm{~mm} \mathrm{Hg}$, entsprechend $15 \%$ des Mittelwertes aus den Messungen des kolloidosmotischen Drucks. Zur Berechnung des Fehlers und Mittelwertes wurden nur die unverdünnten Plasmen herangezogen. Die Abweichung des gemessenen vom berechneten kolloidosmotischen Druck könnte einerseits auf die relative Ungenauigkeit der Messung $\left( \pm 0,5 \mathrm{~cm} \mathrm{H}_{2} \mathrm{O}\right)$, andererseits auf unterschiedliche Konzentration an niedermolekularen Stoffen (Glucose, Triglyceride, Harnstoff) im Plasma zurückzuführen sein. Wie aus früheren Untersuchungen bekannt ist (2), stören diese Stoffe bei der Gesamteiweißberechnung aus der Plasmadichte. Ein weiterer Faktor, welcher Abweichungen zwischen gemessenen und berechneten Werten bedingt, ist die Eiweißzusammensetzung im Plasma. Unterschiedliche Beziehungen zwischen Dichte und kolloidosmotischem Druck bezüglich menschlicher und Hundeplasmen führte Soto-Rivera auf verschiedene Konzentrationen proteingebundener Lipide zurück (11). Neuere Untersuchungen zeigen vor allem einen Einfluß des Eiweißmusters auf die diskutierte Relation (6). Eine entsprechende Abhängigkeit vom Albumin/Globulin-Quotienten bei Humanplasmen konnte auch bei unseren Messungen beobachtet, jedoch nicht statistisch gesichert werden.

\section{Literatur}

1. Hansen, A. T. (1952) Acta Med. Scand. 142, 266.

2. Holzer, H., Leopold, H., Hinghofer-Szalkay, StübchenKirchner, H. \& Maurer, E. (1978) J. Clin. Chem. Clin. Biochem. 16, 391-395.

3. Intaglietta, M. \& Z Weifach, B. W. (1971) Microvasc. Res. 3, $72-82$.

4. Kratky, O., Leopold, H. \& Stabinger, H. (1969) Z. Angew. Phy sik 27, 273-277.

5. Landis, R. M. \& Pappenheimer, J. R. (1963) Exchange of substances through capillary walls. In: Handbook of Physiology: Sect. 2 (Circulation), Vol. II, pp. 961-1034. Am. Physiol. Soc. Washington D.C. 
6. Navar, P. D. \& Navar, L. G. (1977) Am. J. Physiol. 233, 295-298.

7. Prather, J. W., Gaar, K. A. \& Guyton, A. C. (1968) J. Appl Physiol. 24, 602-605.

8. Scatchard, G., Batchelder, A. G. \& Brown, A. (1944) J. Clin. Invest. 23, 459-464.

9. Moore, N. S. \& van Slyke, D. D. (1930) J. Clin. Invest. 8, 337-355.
10. Solenberger, R. I., Jelenko, C. \& Mansberger, A. R. (1977) Am. Surgeon 43, 342-350.

11. Soto-Rivera, A. (1949) Proc. Soc. Exp. Biol. Med. 71, 184-186.

12. Starling, E. H. (1896) J. Physiol. (London) 19, 317.

Max Moser, Dr. H. Hinghofer-Szalkay, Prof. Dr. Th. Kenner

Physiologisches Institut der Universität Harrachgasse 21/5

A-8010 Graz, Austria

Dr. H. Holzer

Medizinische Universitätsklinik

Auenbruggerplatz

A-8036 Graz, Austria 